\title{
Influence of PAN-Fiber Stretching during Thermal Treatment on the Stabilization Reactions
}

\author{
Juliane Meinl', Martin Kirsten², Chokri Cherif², Alexander Michaelis' ${ }^{1}$ \\ ${ }^{1}$ Fraunhofer Institute for Ceramic Technologies and Systems IKTS, Dresden, Germany \\ ${ }^{2}$ Technische Universität Dresden/Institute of Textile Machinery and High Performance \\ Material Technology, Dresden, Germany \\ Email: juliane.meinl@ikts.fraunhofer.de
}

Received 1 February 2016; accepted 13 March 2016; published 16 March 2016

Copyright (C) 2016 by authors and Scientific Research Publishing Inc.

This work is licensed under the Creative Commons Attribution International License (CC BY). http://creativecommons.org/licenses/by/4.0/

(c) (i) Open Access

\section{Abstract}

The stabilization of PAN-fibers without additional co-monomers was investigated with thermogravimetry and evolved gas analysis (FTIR-spectroscopy and MS-spectrometry). One fiber type had been drawn after spinning, while the other was used as-spun. During the thermal treatment, fiber shrinkage was either restricted or unrestricted. Investigations of influencing chemical and physical reactions regarding this restriction were conducted. Differences in the mass loss and gas emissions were observed, depending on the strained or unstrained state of the fibers. The change of crystallinity and molecular orientation of the fiber as reason of the measured variations was discussed. The emission of ammonia and other nitrogen containing gases (supposedly nitriles/ isocyanates) could be attributed to different aspects of the stabilization process. The length restriction resulted in a change in ammonia emission, associated with the cyclization reaction of poly acrylonitrile. The onset and amount of side reactions were influenced as well.

\section{Keywords}

PAN, Polyacrylonitrile Stabilization, Cyclization, Thermogravimetry, Evolved Gas Analysis, Molecular Orientation

\section{Introduction}

Carbon fibers established themselves as a new material class of lightweight high performance fibers for composites [1]-[3]. The majority of today’s carbon fibers are produced from polyacrylonitrile (PAN). The production 
in general consists of two reaction steps: the stabilization step, which is a low temperature heating in air, and a high temperature treatment in inert atmosphere, the carbonization. During the stabilization process, PAN fibers undergo a series of reactions resulting in a material structure suitable for further carbonization treatment. Three main reactions have been established: 1) Cyclization of neighboring nitrile groups leads to the formation of ring structures in the molecule. 2) Dehydrogenation results in the formation of double bonds and (in combination with cyclization) partially aromatic structures mostly conjugate. 3) Oxidation is leading to oxygen functional groups in the arising ladder molecule. The extent to which each of these reactions take place has been discussed in the literature elaborately and several molecular structures of the stabilized PAN have been suggested [4]-[6]. The constitutional setup of those possibilities is shown in Figure 1 representing an overview of the manifold of structural features. The result is a cyclized, partially aromatic structure with oxygen functional groups that does not fuse or degrade in the high-temperature carbonization treatment. Furthermore, side reactions like chain scission as well as degradation have to be taken into consideration regarding the mechanism of structural changes during stabilization, dehydrogenation and oxidation.

The stabilization step is crucial to the quality of the produced carbon fiber. Many parameters have been identified to have an influence on the fiber properties, like heating rate and treatment temperature, gas composition and flow rate, or stretching of the fibers during the stabilization [6]-[10]. The defined drawing of fiber precursors during thermal treatment is an important factor in the industrial carbon fiber synthesis route. The orientation of the PAN molecules during application of a constant tension is maintained and leads to the formation of high-performance carbon fibers with good mechanical properties [11].

Additionally, the draw-ratio of the precursor might influence the reactions during stabilization by enhancing or inhibiting reactions due to steric effects [9] [12]. In polyacrylonitrile fibers, the orientation of the molecules is influenced by the strain, especially the tension during the spinning process. Subsequent drawing of the fibers can further enhance the molecular orientation. A second structural aspect of molecular alignment is the formation of amorphous and crystalline regions in the PAN fiber. The formation of crystalline regions, especially in size as well as in orientation is enhanced by the drawing during the spinning process.

Sen et al. [9] found that an adequate draw ratio of the fiber during the spinning process enhances the ease of dehydrogenation. He suggested that drawing improved the molecular orientation especially in amorphous domains, which could bring more $\mathrm{C}-\mathrm{H}$ groups into a suitable position for dehydrogenation. Besides dehydrogenation, oxidation was influenced as well, while the cyclization seemed to be independent of the drawing process.

A thermal treatment of the precursor fiber leads to two shrinkage processes. First the entropic relaxation of the molecules leads to a contraction of the fiber, second the chemical reactions result in further shrinkage. The molecule then returns to a coiled structure and loses its orientation. Keeping the fibers under tension during the stabilization, the loss of orientation will be reduced. Dalton [13] showed WAXS measurements of fibers with and without tension during heat treatment. Without tension, the loss of orientation visible from the WAXS started at lower temperatures.

Likewise, the entropic relaxation when reheated leads to a reduction of the fiber crystallinity. In several publications, it was assumed that the stabilization reactions are initiated in the amorphous regions of the polymer. Two possible reasons were given: The ease of diffusion of oxygen [14] [15] and the fast initiation of stabilization due to the presence of co-monomers in the amorphous phase, the latter applying only to PAN with a certain co-monomer content [14]. Other scientists concluded that the reaction was not initiated in the amorphous phase, but started throughout the bulk of the material [13].

While the influence of fiber drawing after the spinning process has been investigated before, few publications are available regarding the influence of a stretched status during the thermal treatment. Fitzer [12] assumed from the shrinkage behavior of PAN fibers that under mechanical stress, intramolecular cyclization will be improved. On the other hand Gupta [16] analyzed the mechanical properties of fibers prepared with different stress dur-

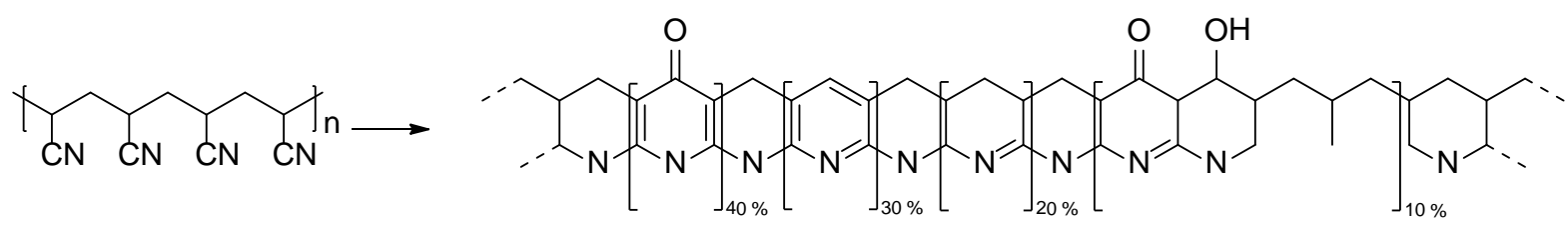

Figure 1. Molecular structure of PAN (left) and overview of constitutional properties of stabilized PAN (right) [7]. 
ing stabilization and proposed, that the rotation of lateral nitrile-groups is inhibited by constraint. In the case of an unhindered shrinkage of a fiber, the rotation of the nitrile groups would then permit more possibilities of intramolecular reactions.

To obtain a defined carbon fiber from a polyacrylonitrile precursor, the addition of co-monomers like methyl acrylates or itaconic acid due to advanced processes in industrial scale has been established [8] [17]. These additives influence the stabilization behavior of the material. Some effects are considered to result from the change in initiation due to the co-monomer content. In many works fiber compositions containing co-monomers are investigated [15] [18] [19], while (especially in newer publications) the reactions of the pure homopolymer PAN are rarely considered.

A deeper understanding of the material and process development can only be realized by taking all the ongoing reactions into account. A lot of work on stabilization has been published, and still questions concerning the order and extent of certain reactions as well as the mechanisms of structural changes remain unclear.

This work aims to study the influence of fiber stretching during the thermal treatment by fixing the samples during the thermal analysis. Additionally, evolved gas analysis is coupled to the thermogravimetric measurements to gain information about individual gas emissions, which will allow for a differentiation of reactions during the stabilization process.

\section{Experimental}

Two types of PAN fibers (fiber 1 and 2), consisting of the same polymer but prepared with absolutely different processing parameters were chosen in order to conduct the investigations. Both were spun via solution spinning of PAN using Dimethylformamide (DMF) as solvent. The spinning was conducted on a wet-spinning pilot plant by Fourné Polymertechnik GmbH, Germany consisting of one coagulation (solvent composition DMF 85\%, water $15 \%$ ), three washing baths, one drying and sizing unit and a winder with an overall speed of up to 60 $\mathrm{m} / \mathrm{min}$. Fiber 2 was additionally drawn after spinning. This was performed on a pilot plant scale post-drawing unit by DIENES Apparatebau GmbH, Germany where the yarn is transported and heated by duos of godets. The heat was transferred by direct contact into the fiber. The draw ratio during the wet-spinning and post-drawing was set to 10.9 for the overall process of fiber formation. The fiber properties are listed in Table $\mathbf{1}$. Fiber $\mathbf{1}$ is characterized by a loose character having a cross section diameter of $40 \mu \mathrm{m}$, a tenacity of $13 \mathrm{cN} /$ tex and an elastic modulus of $3.3 \mathrm{cN} /$ tex. The fiber is poorly stretched having a draw ratio of 4.3 regarding the overall process. The properties of fiber 2 are more suitable for an industrial approach of the conversion to carbon fibers by thermal treatment. A single filament cross section of $13.8 \mu \mathrm{m}$, a tenacity of $42 \mathrm{cN} / \mathrm{tex}$ and an elastic modulus of $16.1 \mathrm{cN} /$ tex were found.

Mass change is an essential element of the stabilization process in polyacrylonitrile and thermogravimetric analysis is a useful tool for its investigation. By coupling with evolved gas analysis, information about specific reactions and their resulting emissions becomes available and can be compared with the overall thermal behavior. It helps in distinguishing the diverse reactions of the complex process of PAN stabilization.

Thermal Gravimetric Analysis (TGA) was carried out with a STA 449 F1 (NETZSCH-Gerätebau GmbH). The gas outlet was coupled with Evolved Gas Analysis by FTIR-Spectroscopy and Mass Spectrometry. The FTIR-spectroscope was a Bruker Optics Tensor 27 and was connected by a heated transfer line to the TGA. The MS was a quadrupole mass spectrometer QMS 403C (Netzsch) which was connected via a capillary coupling system.

The fixed samples were fixed on an alumina cylinder (Figure 2), to inhibit the shrinkage during thermal treatment and placed in a large alumina crucible that allowed good heat and gas exchange. The loose samples remained loosely in the large alumina crucible.

Table 1. Properties of the prepared fibers.

\begin{tabular}{ccc}
\hline Parameter & Fiber 1 & Fiber 2 \\
\hline Draw ratio & 4.3 & 10.9 \\
Diameter & $40 \mu \mathrm{m}$ & $13.8 \mu \mathrm{m}$ \\
Tenacity & $13 \mathrm{cN} / \mathrm{tex}$ & $42 \mathrm{cN} / \mathrm{tex}$ \\
Elastic modulus & $3.3 \mathrm{cN} / \mathrm{tex}$ & $16.1 \mathrm{cN} / \mathrm{tex}$ \\
\hline
\end{tabular}




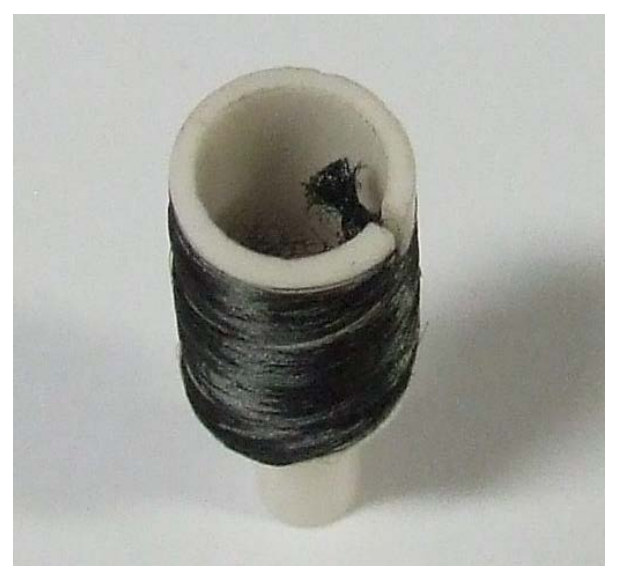

Figure 2. Fixed fiber sample on alumina cylinder after thermal treatment.

The experiments were conducted in air atmosphere with a flow rate of $5 \mathrm{l} / \mathrm{h}$. A heating rate of $2 \mathrm{~K} / \mathrm{min}$ was used to study the reactions during the stabilization. Due to the exothermal nature of the stabilization, the reactions in PAN fibers are very sensitive to the heating rate. In the industry, low heating rates are used to prevent the fibers from overheating leading to massive fiber damage and to minimize degradation reactions. Higher heating rates can accelerate side reactions and weaken the fiber structure. Nevertheless, the interest to shorten process times is immanent. From a scientific view, the reaction characteristics under different conditions can give additional information. Therefore a second temperature program with a heating rate of $5 \mathrm{~K} / \mathrm{min}$ was also used for one fiber type (fiber 1). The two heating rates were chosen based on preliminary investigations.

Although the FTIR spectroscopy was not performed focusing on quantitative analysis, a semi-quantitative comparability is realized by identical measurement conditions and sample sizes. FTIR-spectra of the gas emissions were recorded during the thermal treatment. Characteristic peaks were assigned to gaseous emissions and the respective peak integral was calculated. The resulting FTIR-trace is the FTIR-signal shown in the measurements. Additionally, the FTIR-signal was weighted according to the sample mass, to account for small differences in sample weight.

For additional fiber characterization, thermomechanical analysis was done with a TMA 402 (Netzsch) and caloric effects of the samples were measured with a DSC 404 (Netzsch).

\section{Results}

The results from thermogravimetric analysis will be discussed first, showing the overall behavior of mass change of the fibers and the different fiber preparations. Second, certain aspects of the reaction processes will be further analyzed by the results from evolved gas analysis.

\subsection{Thermogravimetry}

The samples show some mass loss at lower temperatures due to residual water or DMF originating from the spinning solution. This is especially visible in fiber 2 (Figure 3). The main mass loss resulting from stabilization reactions begins at temperatures above $200^{\circ} \mathrm{C}$. It can be observed up to $325^{\circ} \mathrm{C}$ and is followed by a subsequent mass loss at a lower rate up to the maximum temperature of the thermal treatment.

At a heating rate of $2 \mathrm{~K} / \mathrm{min}$ (Figure 3) the mass change rate shows a peak maximum around $280^{\circ} \mathrm{C}$. A second smaller peak, visible as a shoulder in the range of $290^{\circ} \mathrm{C}$ to $330^{\circ} \mathrm{C}$, can be determined. The mass loss and mass change rate up to $300^{\circ} \mathrm{C}$ in fiber 2 is higher than in fiber 1 , which would represent a larger amount of stabilization reactions in the material. Comparing the fixed and loose condition, the loose fiber, the peak maximum is higher, while the shoulder is less prominent than in the fixed fiber. Onset and total mass loss differ only slightly and the peak mass change rate is shifted to slightly higher temperatures in the fixed fibers.

The reactions during stabilization are influenced by the heating rate. The cyclization is known to be an exothermal reaction, which can even lead to a local overheating in the fiber that enhances scission and degradation of the polymer backbone. Exemplary DSC measurements of fiber 1 are shown in Figure 4, similar results were 

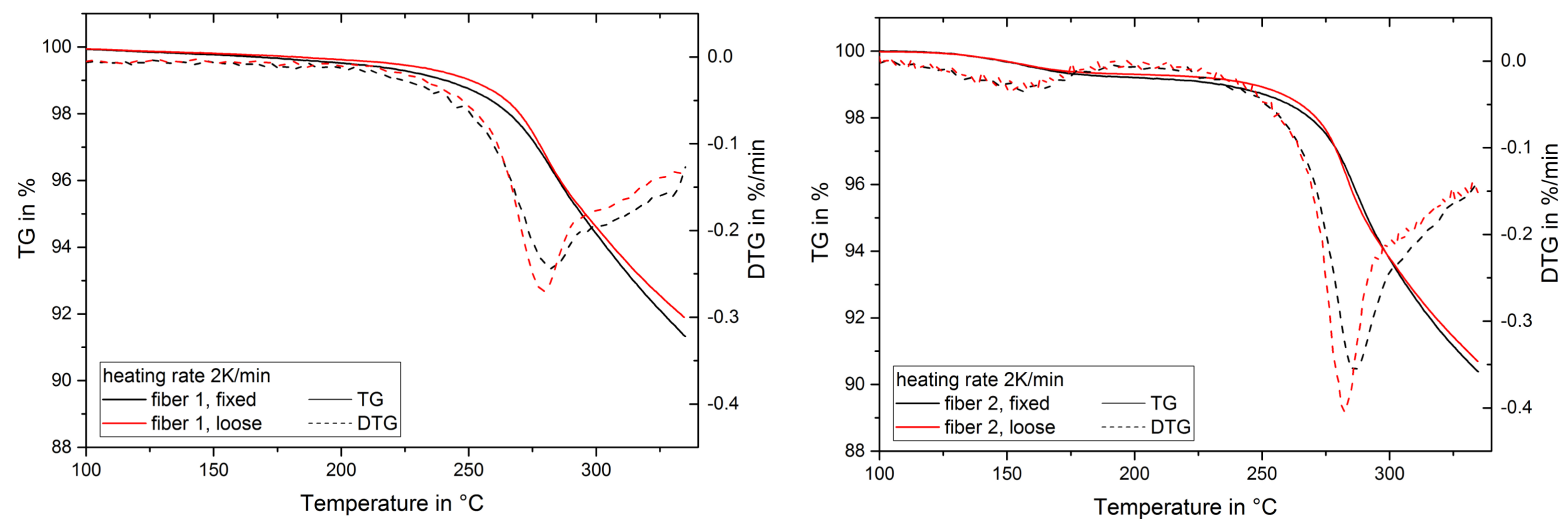

Figure 3. Mass loss (TG) and mass change rate (DTG) during thermal treatment of fiber 1 (left) and 2 (right) at $2 \mathrm{~K} / \mathrm{min}$.
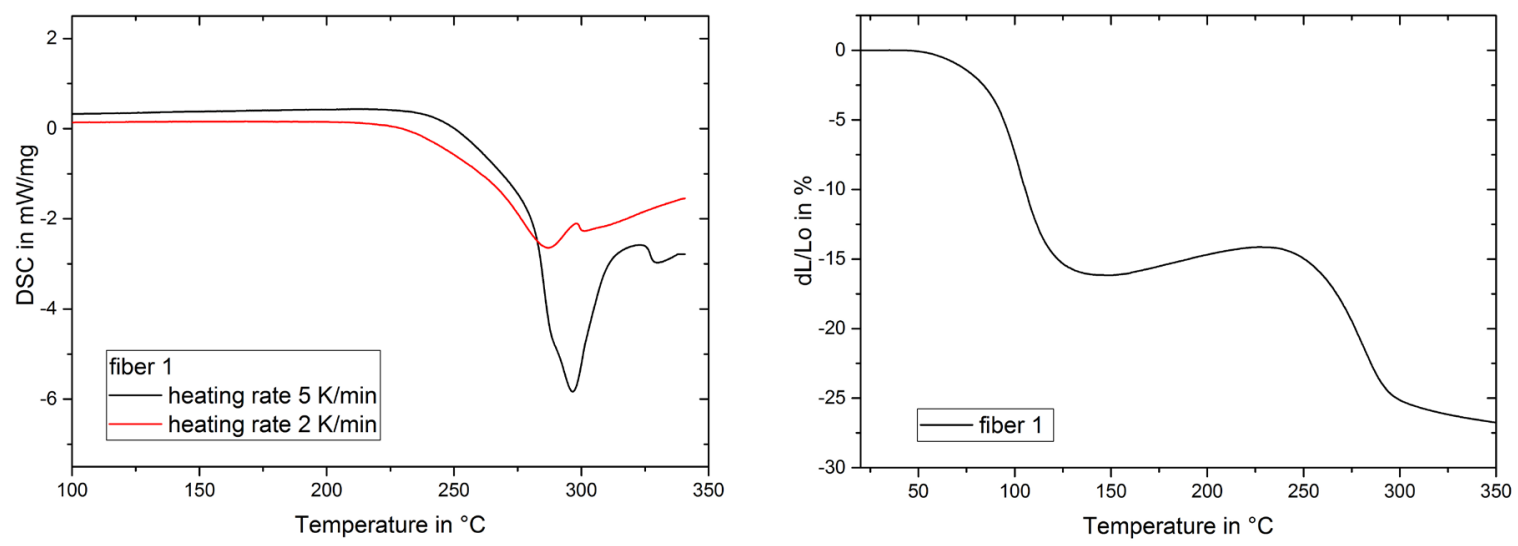

Figure 4. Left: DSC-signal of fiber 1 during thermal treatment in air. Right: Length change of fiber 1 during thermal treatment in air at low load.

found for fiber 2 as well. The exothermal effect during stabilization appears stronger in fibers at higher heating rates.

The fixed fibers appear undamaged after the thermal treatment at a heating rate of $2 \mathrm{~K} / \mathrm{min}$, while at $5 \mathrm{~K} / \mathrm{min}$ a tear in the fixed fiber can be seen. In TMA experiments at low load, the strong shrinkage of fibers during heating and stabilization can be shown to reach values of more than 25\% (Figure 4). The two stages of shrinkage, physical shrinkage from entropic relaxation $\left(50^{\circ} \mathrm{C}-150^{\circ} \mathrm{C}\right)$ and chemical shrinkage from stabilization $\left(225^{\circ} \mathrm{C}-300^{\circ} \mathrm{C}\right)$, can be clearly distinguished. Since the crack size of the strained fibers is very small, one can assume that the tear occurred very late during the stabilization process and that the fiber remained under strain for most of the experiment.

At a heating rate of $5 \mathrm{~K} / \mathrm{min}$ a strong difference in the mass loss of strained and loose fibers can be observed (Figure 5). In fixed fibers the onset of mass loss and the peak mass change rate (DTG) are shifted to higher temperatures, which is the same tendency as in the $2 \mathrm{~K} / \mathrm{min}$ samples, but more pronounced. The total mass loss is lower and the mass change rate shows two peaks at $284^{\circ} \mathrm{C}$ and $296^{\circ} \mathrm{C}$ instead of one peak at $280^{\circ} \mathrm{C}$ in the DTG signal of the loose fiber. This second peak might result from the same reactions as the shoulder seen in the 2 $\mathrm{K} / \mathrm{min}$ experiments in Figure 3, but in contrast the shoulder there appears in both loose and fixed fibers. Again a subsequent mass loss up to the maximum temperature continues.

In summary, all thermogravimetric experiments show a change in mass loss, where the maximum mass change rate is lower and the peak temperature is shifted to higher temperatures in the fixed fiber. The effect is more prominent at higher heating rates, where the form of the DTG-peak changes as well and the mass loss is lower for the fixed fibers. Since all other parameters remained the same during the thermoanalytic investigation, the cause for the differences of fixed and loose fibers in mass loss would be caused by the strained or unstrained state of the fibers. As mentioned before, the fiber will lose orientation and crystallinity due to entropic relaxation, 


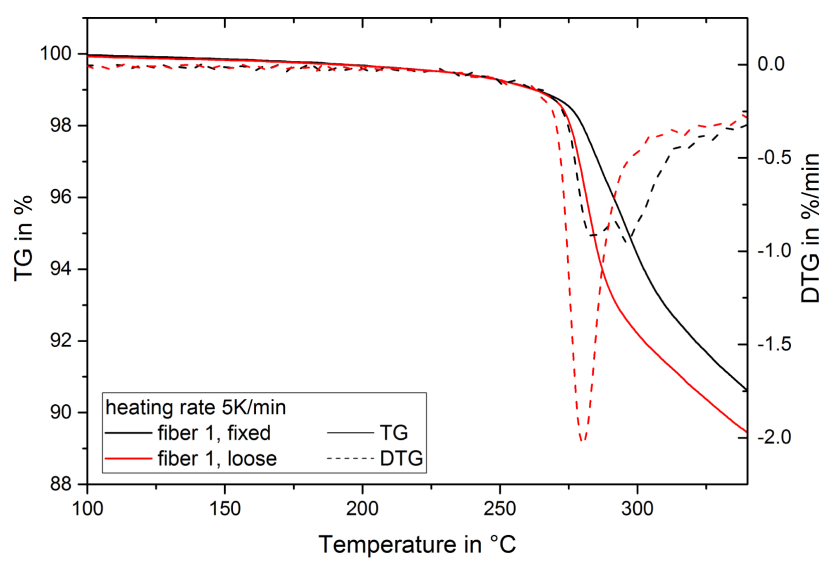

Figure 5. Mass loss (TG) and mass change rate (DTG) during thermal treatment of fiber 1 at $5 \mathrm{~K} / \mathrm{min}$.

if it is not kept in a strained state during heating. As can be seen in the TMA measurements, the physical shrinkage, which leads to a loss of orientation and crystallinity, takes place before the stabilization reactions. Therefore the molecular orientation might play the critical role in explaining the significant differences in the thermal investigation. For additional information results from emission gas analysis are taken into consideration.

\subsection{Evolved gas Analysis}

During the stabilization process a number of gaseous products like $\mathrm{CO}_{2}, \mathrm{CO}, \mathrm{H}_{2} \mathrm{O}$, hydrocarbons and several nitrogen-containing compounds (e.g. ammonia, hydrogen cyanide, isocyanates, nitriles) are produced. Figure 6 gives an overview of gaseous compounds detected by FTIR-spectroscopy during the thermal treatment.

Corresponding to the mass loss, the main gas emissions start at temperatures above $200^{\circ} \mathrm{C}$ and reach a maximum in the range of the strongest mass loss of both samples. In Figure 7 the development of emissions of different gas components detected by FTIR and MS is shown exemplary for fiber 1 . Although many gases have a peak of emission at the maximum mass loss of $280^{\circ} \mathrm{C}$, the emission characteristics of each gas component show distinct differences.

The signal of $\mathrm{H}_{2} \mathrm{O}$ - and HCN-emissions shows a strong resemblance to the DTG-signal of the thermogravimetry (Figure 3). It can be concluded, that they represent a large fraction of the mass loss during stabilization. Ammonia shows a strong peak as well, with the same maximum as $\mathrm{H}_{2} \mathrm{O}$ and $\mathrm{HCN}$, but no shoulder at higher temperatures can be detected, which means that at least two different reactions lead to the mass loss during stabilization. Nitriles and isocyanates show two peaks of emission and the temperature of the second peak maximum is situated at a temperature, where no corresponding peak in the DTG-signal can be seen. Therefore their fraction of the total mass loss is small. $\mathrm{CO}_{2}$ and acrylonitrile monomer emissions continue to the maximum temperature and therefore contribute to the subsequent mass loss at temperatures above $300^{\circ} \mathrm{C}$. At a heating rate of $2 \mathrm{~K} / \mathrm{min}$, alkanes were not detected in significant amounts.

\subsubsection{Cyclization and Ammonia Emission}

Ammonia emission is often assigned to the cyclization reaction. Theoretically, cyclization would not lead to any mass loss, but the reaction is initiated at several points in the molecule and proceeds only for a number of functional groups along the polymer backbone. Imine groups were suggested as terminal structures of such a cyclized segment. Further reaction of the imine groups or remaining nitrile groups can then lead to ammonia emissions [9] [20]. Therefore cyclization and ammonia emission are correlated.

In contrast to the other gas emissions in our experiments, the ammonia emission appears as a single peak without any strong shoulder or overlapping during the temperature treatment at $2 \mathrm{~K} / \mathrm{min}$ (Figure 8). The maximum of the ammonia emission appears slightly before the maximum of the DTG-signal. The higher peak in the loose fibers accounts for a higher ammonia emission.

During the $5 \mathrm{~K} / \mathrm{min}$ heat treatment a shoulder can be seen in the ammonia emission of the fixed fibers (Figure 9). It corresponds to the DTG-signal, which shows two peaks instead of one. This is contrary to the shoulder 


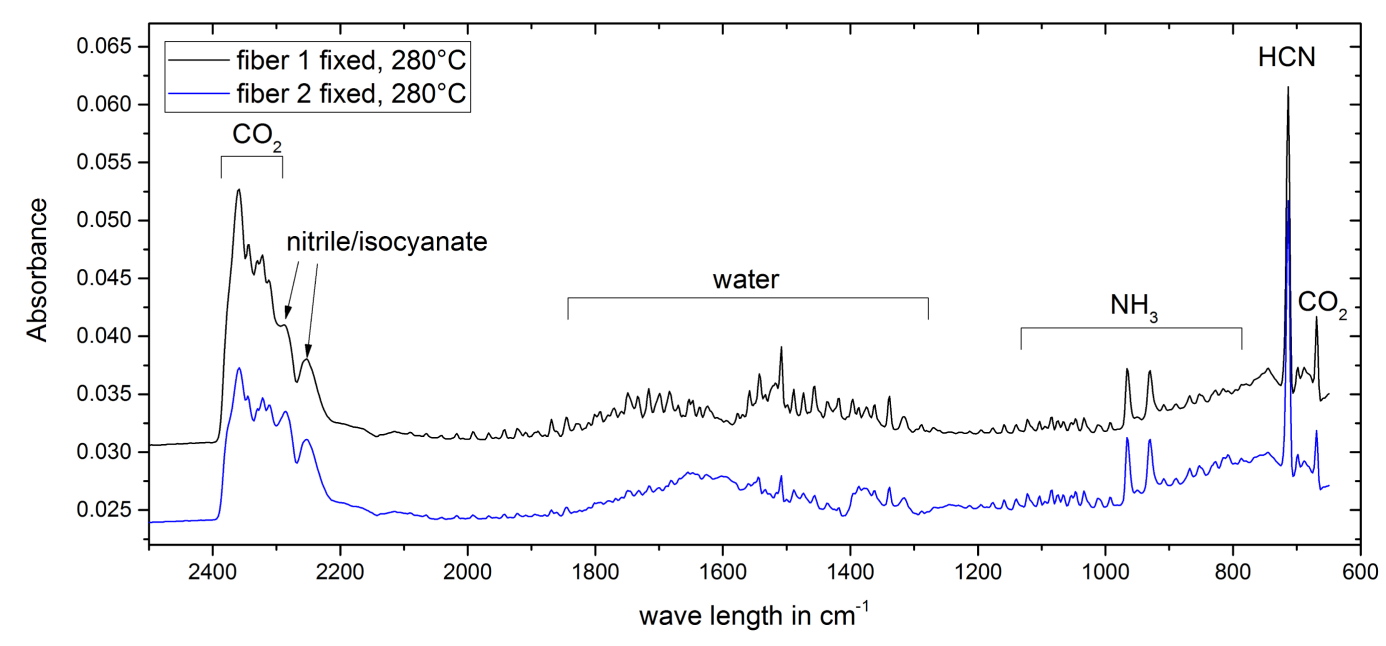

Figure 6. FTIR-spectrum of gas emissions from fiber 1 and 2 at $280^{\circ} \mathrm{C}$ during thermal treatment with $2 \mathrm{~K} / \mathrm{min}$.

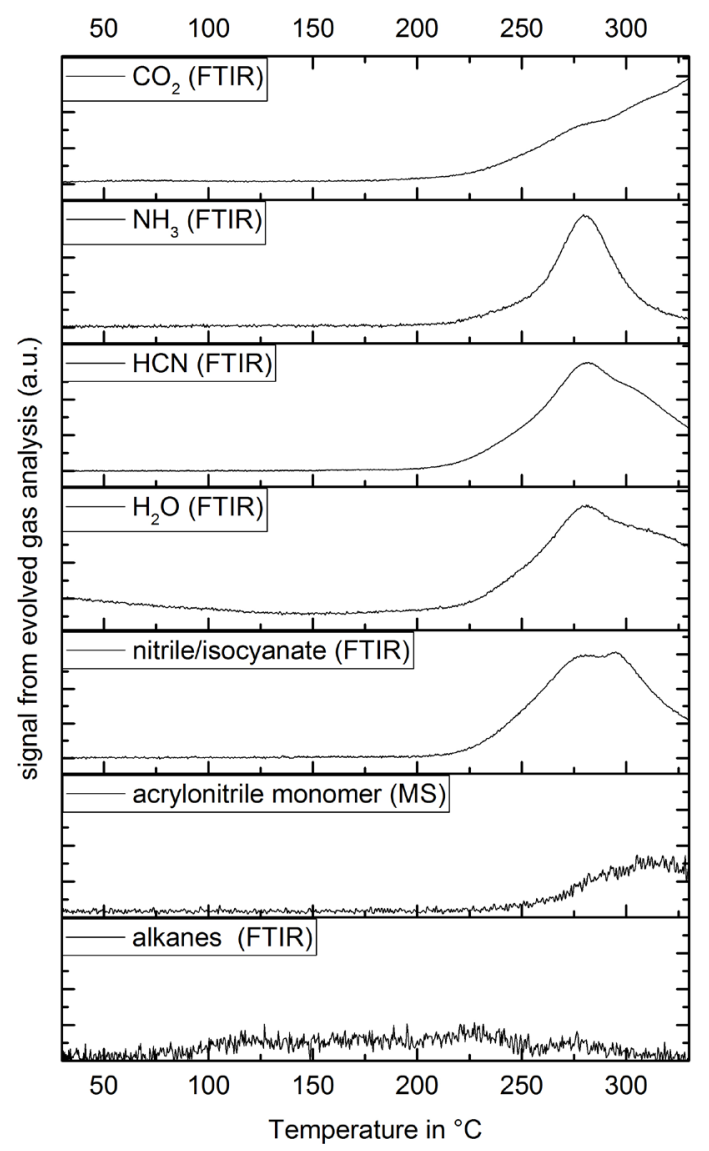

Figure 7. Overview of gas emissions from fiber 1 (fixed) during thermal treatment at $2 \mathrm{~K} / \mathrm{min}$.

seen in the DTG of $2 \mathrm{~K} / \mathrm{min}$ experiments, which is not accompanied by corresponding ammonia emissions. The split into two peaks at $5 \mathrm{~K} / \mathrm{min}$ could account for two parallel reactions resulting in ammonia release, which are overlapped closely when the fiber is heated at $2 \mathrm{~K} / \mathrm{min}$.

The ammonia formation is much stronger in loose fibers. Two opposite assumption can be made: 1) the ammonia formation arises from terminal imine groups produced during stabilization. A lower ammonia release could be the result of longer cyclization propagation with less interruptions by terminal structures, which is 

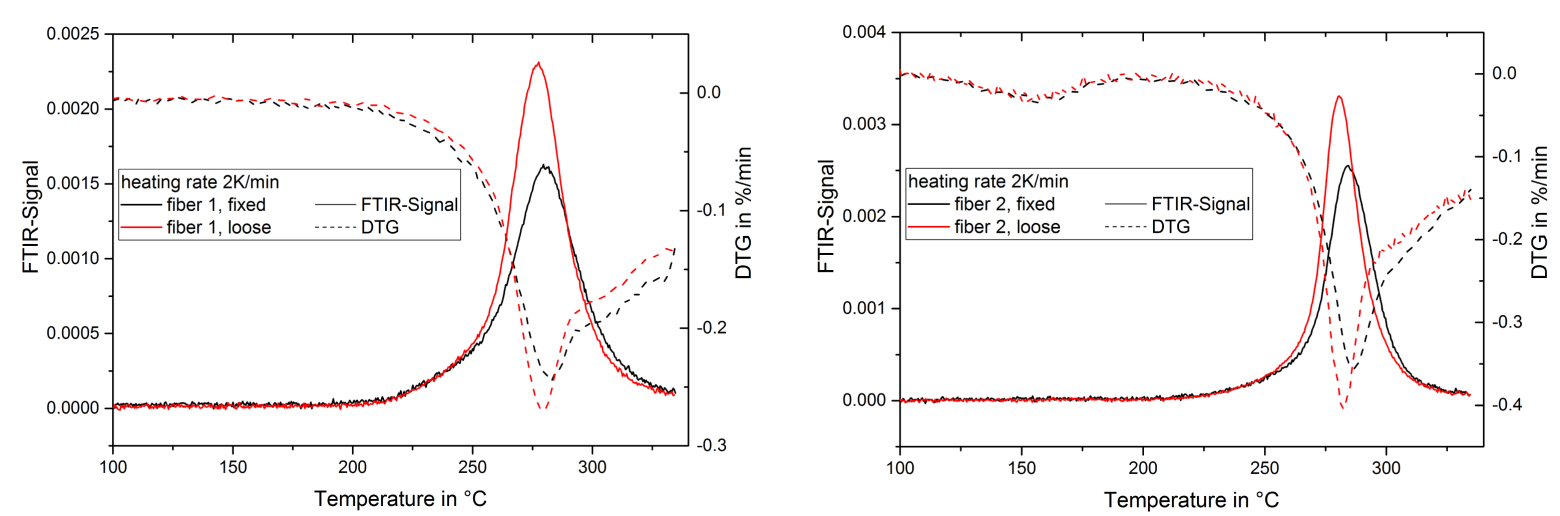

Figure 8. FTIR-signal of ammonia in gas emission and mass change rate (DTG) during thermal treatment of fiber 1 (left) and 2 (right) at $2 \mathrm{~K} / \mathrm{min}$.

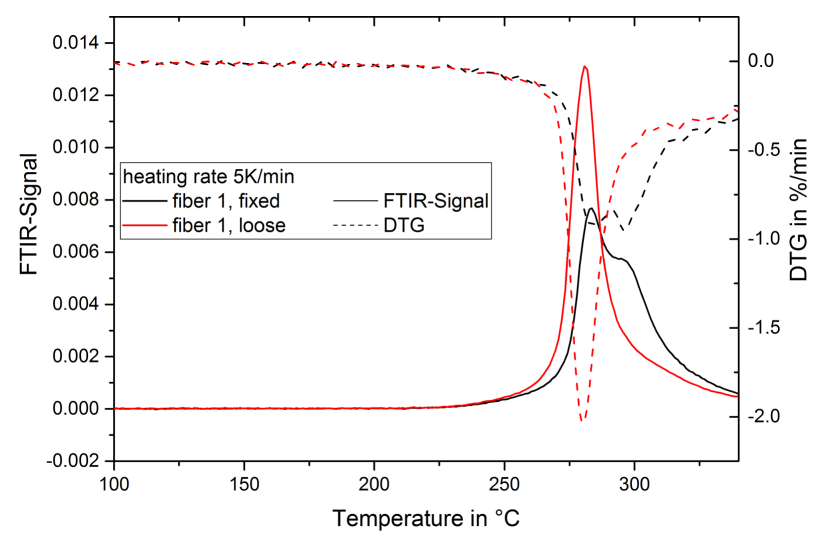

Figure 9. FTIR-signal of ammonia in gas emission and mass change rate (DTG) during thermal treatment of fiber 1 at 5 $\mathrm{K} / \mathrm{min}$.

beneficial to the molecular ladder formation. This would be in accordance with assumptions from Fitzer [12]. 2) The cyclization in general is hindered in strained fibers (e.g. stereochemically). Comparing the two different fibers, the ammonia emission in fiber 2 is stronger and the peak is narrower than in fiber 1 . This corresponds to the DTG-peak in the same temperature range, which shows the same effect. Fiber 2 had been spun and drawn with optimized conditions for a carbon fiber precursor. It has a smaller diameter than fiber 1, which improves heat dissipation and diffusion processes in the fiber cross section and has a higher degree of orientation due to post-drawing. This improves the stabilization reactions and hence leads to a higher ammonia emission.

If the ammonia formation is caused by reactions of terminal imine groups, the cyclization has already advanced throughout the structure at the point of ammonia emission. This would suggest that further reactions are not part of cyclization, but of other reactions like crosslinking or oxidation.

Besides ammonia, cyanic acid (HCN) is referred to as a main volatile during the cyclization reactions [21] [22], but as can be seen from the evolved gas analysis in Figure 7, the ammonia and HCN emissions differ in its behavior and characteristics. The emission of HCN corresponds strongly with the mass change rate signal. The peak temperatures are the same and a shoulder is visible at higher temperatures. A similar behavior can be seen in water emissions. The HCN emission shows a peak at a similar temperature as ammonia, but a much broader peak is underlying, starting at lower temperatures and visible as a shoulder at higher temperatures. While the concurrent peak of HCN and ammonia might result from the same reaction (supposedly cyclization), the broad peak could be the result of degradation. The influence of strain on the HCN emission is much smaller. The formation of water can be attributed to dehydrogenation reactions as well as degradation. It shows some similarities to HCN and can similarly be viewed as two peaks, one broad peak which is overlapping with a narrower peak. If the broad peak is a result of degradation as well, the narrow peak might be caused by dehydrogenation, implying that dehydrogenation and cyclization are concurrent reactions in the stabilization process. 


\subsubsection{Gas emissions from Side Reactions}

Side reactions during the stabilization process lead to degradation of the material, weakening of the material structure and reduction of the carbon yield. Typical emissions from side reactions are $\mathrm{CO}_{2}$, hydrocarbons and different organic substances containing nitrogen functionalities.

In our experiments, FTIR-signals were found in the wave numbers from 2300 to $2230 \mathrm{~cm}^{-1}$. They are partially overlapped by the IR-bands of $\mathrm{CO}_{2}$, but their progress over the heating program is very distinct and differs strongly from $\mathrm{CO}_{2}$. The peaks were assigned to the $\mathrm{C} \equiv \mathrm{N}$ or $\mathrm{N}=\mathrm{C}=\mathrm{O}$ stretching vibrations. The exact composition of these gaseous products could not be completely identified. The monomer acrylonitrile does not show a strong peak in this wavenumber region and by comparison with the data of mass spectrometry it could be ruled out as the source of this gas emission. Methylisocyanate or other short isocyanates could be the cause as well as different nitrile compounds. In decomposition studies of PAN [23] a number of different nitriles were found to result from the thermal treatment of the material.

Two peaks are visible, the first of which corresponds with the temperature of the maximum mass change rate at $280^{\circ} \mathrm{C}$ while no corresponding DTG-peak can be found for the second peak of the FTIR-signal at $295^{\circ} \mathrm{C}$ (Figure 10). The first peak is more prominent in loose fibers, while the second peak appears stronger in fixed fibers.

The FTIR-signal shows a stronger emission of nitriles and isocyanates in fiber 2 than in fiber 1. Again, as mentioned for the ammonia emissions before, fiber 2 had been optimized for the carbon fiber process and therefore shows an increased amount of stabilization reactions compared to fiber 1. Since the nitrile/isocyanate emissions increase as well, there are also more side reactions during stabilization. Further investigations would be necessary to quantify, if the overall ratio of intended stabilization reactions (cyclization, dehydrogenation, oxidation) to side reactions is still improved.

At $5 \mathrm{~K} / \mathrm{min}$ heating rate the peaks are more clearly separated (Figure 11). The second peak is shifted to $315^{\circ} \mathrm{C}$
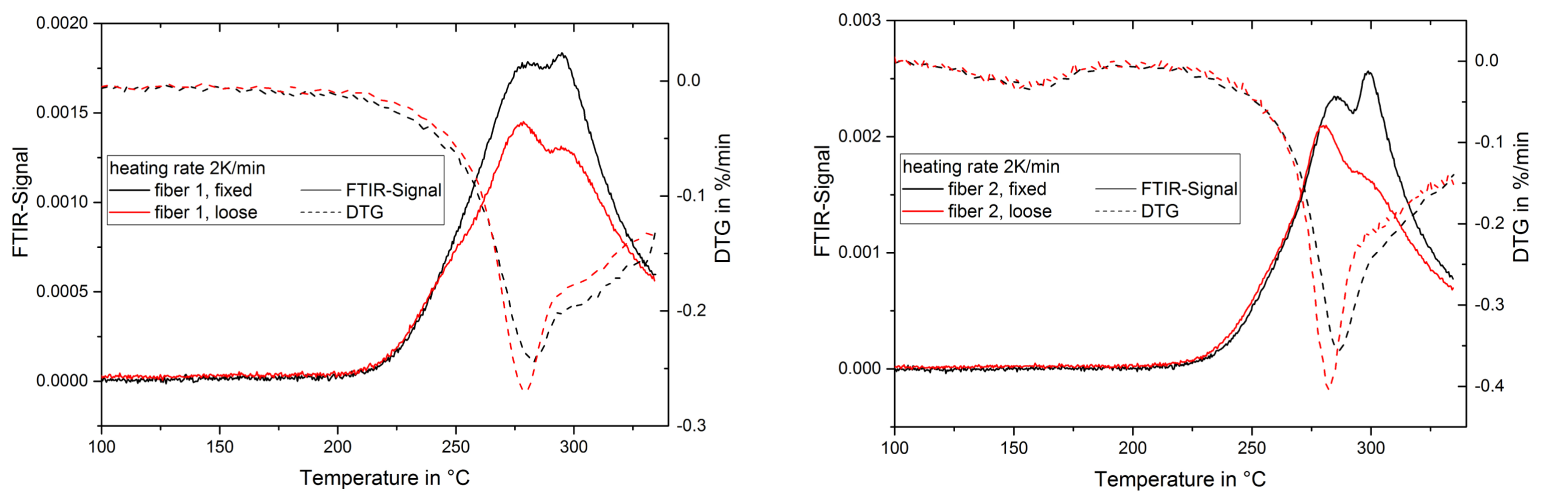

Figure 10. FTIR-signal of nitrile/isocyanate groups in gas emission and mass change rate (DTG) during thermal treatment of fiber 1 (left) and 2 (right) at $2 \mathrm{~K} / \mathrm{min}$.

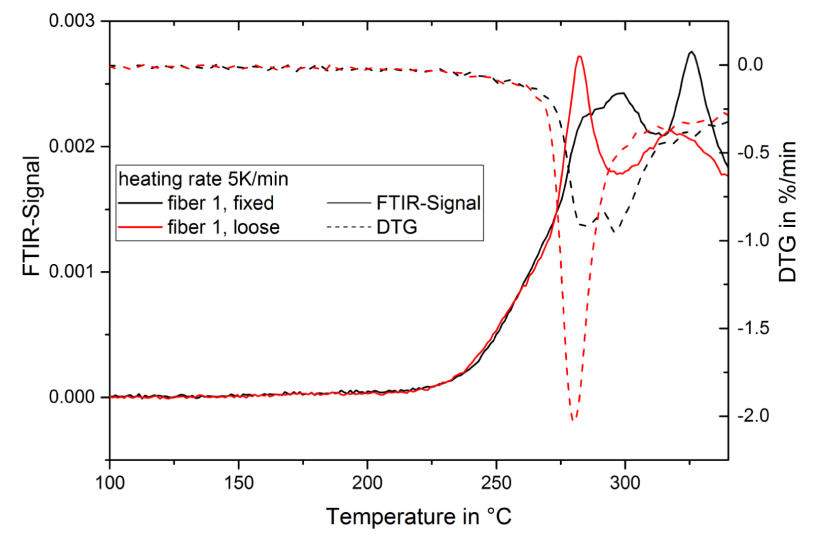

Figure 11. FTIR-signal of nitrile/isocyanate groups in gas emission and mass change rate (DTG) during thermal treatment of fiber 1 at $5 \mathrm{~K} / \mathrm{min}$. 
in the loose fiber and $325^{\circ} \mathrm{C}$ in the fixed fiber. Additionally, the first peak is split into two peaks at 283 and $297^{\circ} \mathrm{C}$ in strained fibers. This split of the peak is comparable to the ammonia emission (Figure 9) and the overall mass change rate at $5 \mathrm{~K} / \mathrm{min}$ in the fixed fiber.

In the literature, it was proposed that stabilization reactions might occur at lower temperatures in amorphous parts of the polymer and at higher temperatures in the crystalline parts [14] [15]. This would be a possible explanation for the two peaks seen here. In a fixed state, the ratio of crystalline regions is higher and the first peak is reduced while the second peak increases. The peaks are separated more clearly in fiber 2, which underwent post- drawing resulting in a higher orientation of the polymer chains into fiber direction. Especially the second peak in the fixed fiber 2 appears stronger, which could be explained by the higher crystallinity. Regarding the origin of the gases containing nitrile or isocyanate functional groups, they probably result from chain scission processes in the fiber. In crystalline regions of the fiber, the interaction of the nitrile groups in PAN leads to a stabilization of the structure, which would explain a higher onset temperature for degradation processes.

As mentioned before, it has been assumed by Gupta [14], that the co-monomer content leads to a preferred onset of the stabilization reactions in the amorphous phase. Assuming the change in nitrile emission to result from amorphous and crystalline regions, it implies that similar differences in the onset of reactions exist in a co-monomer free material as well.

Other common gas emissions of degradation and chain scission are $\mathrm{CO}_{2}$, acrylonitrile and hydrocarbons. $\mathrm{CO}_{2}-$ emissions increase during the stabilization reaction up to the end of the thermal treatment. A further temperature increase in air would naturally lead to the complete combustion of the material. It should be kept low to prevent a decrease in the carbon yield and the destruction of the fiber structure. During the steady increase of $\mathrm{CO}_{2}$ there are two slight peaks visible at $2 \mathrm{~K} / \mathrm{min}$, the first can be assigned to the mass loss maximum while the second appears during the shoulder in the range of $290^{\circ} \mathrm{C}$ to $330^{\circ} \mathrm{C}$ (Figure 12). The second peak corresponds with the HCN emission, both products might therefore result mainly from degradation at this point

At $5 \mathrm{~K} / \mathrm{min}$ heating rate, there is a strong $\mathrm{CO}_{2}$-peak at $280^{\circ} \mathrm{C}$ in the loose fiber which was clearly reduced in the fixed fiber (Figure 13). Similar characteristics could be found in the formation of hydrocarbons and acrylonitrile monomer (Figure 14). Again, the continued stretching during stabilization provides an inhibiting effect on side reactions. During the experiments at $2 \mathrm{~K} / \mathrm{min}$ monomer emission was only detected at a very low intensity and no other hydrocarbons were visible.

\section{Conclusions}

The presented measurements show the complexity of chemical reactions as well as physical changes during the stabilization process of polyacrylonitrile. The reactions of PAN fibers under stabilization conditions were studied by thermogravimetry and coupled evolved gas analysis. A sample preparation allowing for shrinkage limitation during the analysis was used, to compare loose and fixed fibers and proved to show distinct differences in the thermal behavior. While some publications have discussed the influence of stretching during the thermal treatment based on fiber shrinkage and mechanical properties, no literature existed yet investigating the influence of strain during the thermal treatment by thermogravimetry and study of gas emissions.
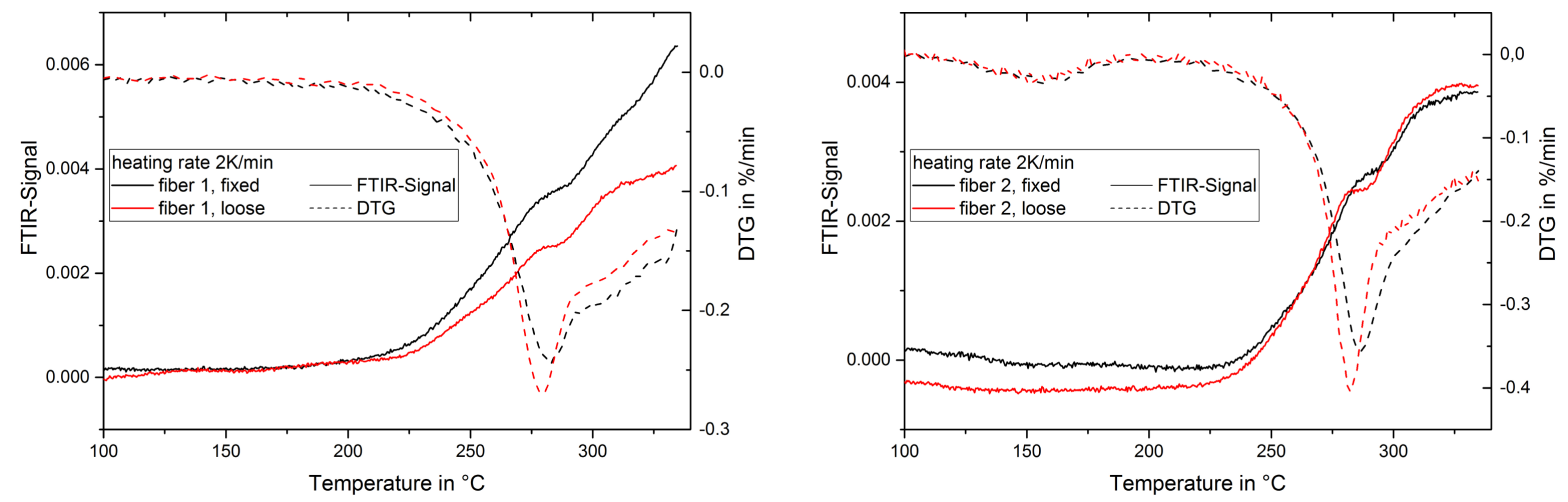

Figure 12. FTIR-signal of $\mathrm{CO}_{2}$ in gas emission and mass change rate (DTG) during thermal treatment of fiber 1 (left) and 2 (right) at $2 \mathrm{~K} / \mathrm{min}$. 


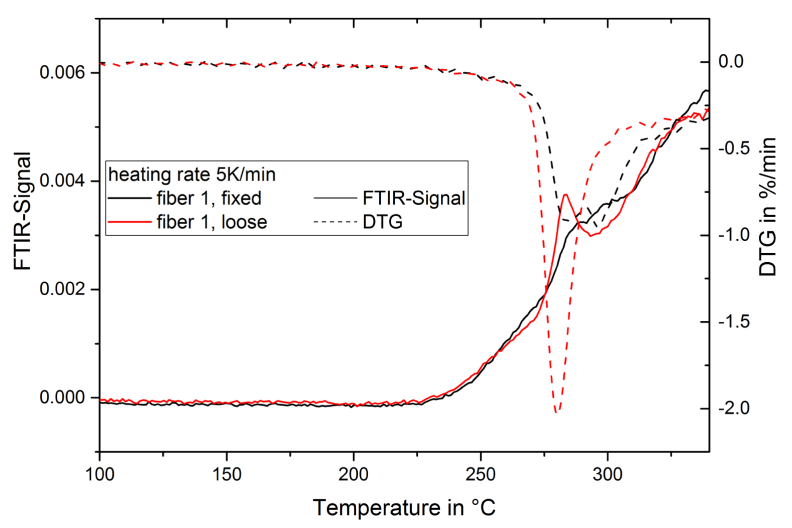

Figure 13. FTIR-signal of $\mathrm{CO}_{2}$ in gas emission and mass change rate (DTG) during thermal treatment of fiber 1 at $5 \mathrm{~K} / \mathrm{min}$.
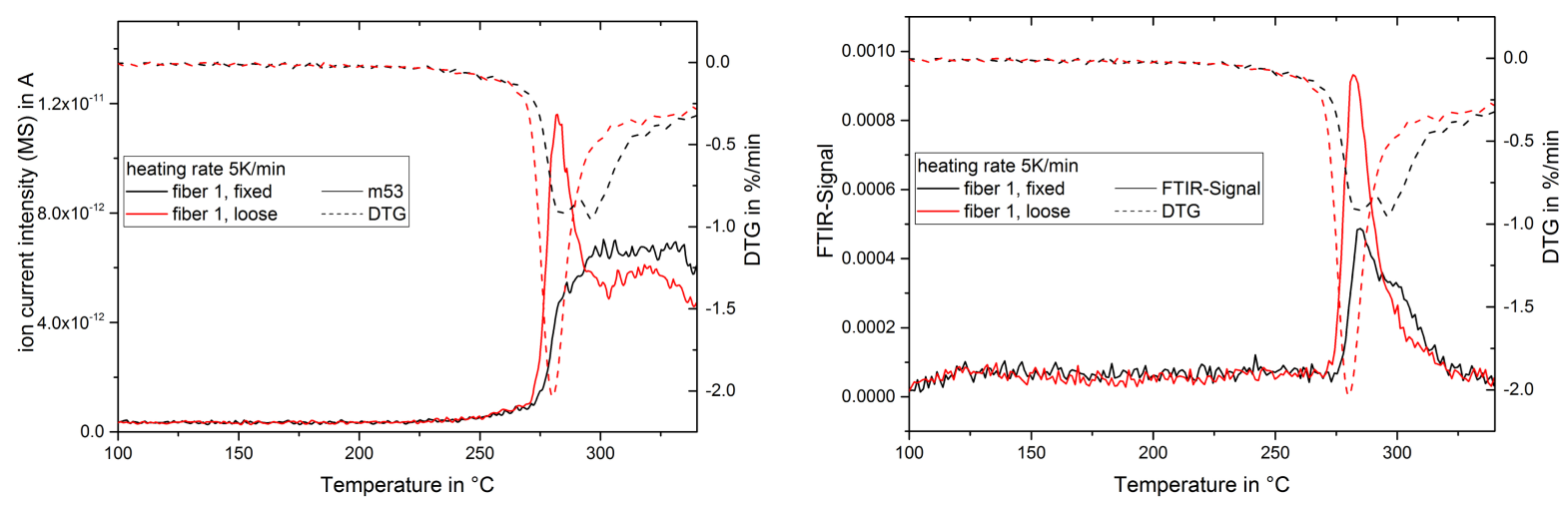

Figure 14. MS-signal of acrylonitrile monomer (left)/FTIR-signal of alkane (right) in gas emission and mass change rate (DTG) during thermal treatment of fiber 1 at $5 \mathrm{~K} / \mathrm{min}$.

Two aspects were regarded: the main cyclization and dehydrogenation reactions and the side reactions from scission and degradation. The emission of ammonia and HCN could be attributed to cyclization reaction, but it was shown that HCN is also a volatile product of further reactions apart from cyclization. The intensity of the ammonia emission changes significantly between loose and fixed fibers. The influence of orientation during thermal treatment on the cyclization can be seen, but based on the ammonia emissions, no indications were found for different reaction onsets in amorphous and crystalline regions of the polymer.

On the other side, the nitrile and isocyanate emissions were associated to side reactions and appeared as a double peak that could be explained to result from different reaction onsets in amorphous and crystalline regions of the material. A stabilizing effect of molecular orientation on the fiber is especially visible in the reduction of side reaction.

Further investigations are ongoing to obtain more knowledge about the certain physical and chemical processes which are taking place at the same time and overlapping as well as influencing itself during the stabilization.

\section{Acknowledgements}

The authors gratefully acknowledge the financial support by the European Union (EFRE) and Freistaat Sachsen (SAB EFRE 100062605).

\section{References}

[1] Fitzer, E., Kleinholz, R., Tiesler, H., Stacey, M.H., et al. (2000) Fibers, 5. Synthetic Inorganic. In: Ullmann’s Encyclopedia of Industrial Chemistry, Wiley-VCH Verlag GmbH \& Co. KGaA, Weinheim.

[2] Huang, X. (2009) Fabrication and Properties of Carbon Fibers. Materials, 2, 2369-2403.

http://dx.doi.org/10.3390/ma2042369 
[3] Zhang, J., Terrones, M., et al. (2016) Carbon Science in 2016: Status, Challenges and Perspectives. Carbon, 98, 708732. http://dx.doi.org/10.1016/j.carbon.2015.11.060

[4] Bashir, Z. (1991) A Critical Review of the Stabilisation of Polyacrylonitrile. Carbon, 29, 1081-1090. http://dx.doi.org/10.1016/0008-6223(91)90024-D

[5] Wangxi, Z., Jie, L. and Gang, W. (2003) Evolution of Structure and Properties of PAN Precursors during Their Conversion to Carbon Fibers. Carbon, 41, 2805-2812. http://dx.doi.org/10.1016/S0008-6223(03)00391-9

[6] Yusof, N. and Ismail, A. (2012) Post Spinning and Pyrolysis Processes of Polyacrylonitrile (PAN)-Based Carbon Fiber and Activated Carbon Fiber: A Review. Journal of Analytical and Applied Pyrolysis, 93, 1-13. http://dx.doi.org/10.1016/j.jaap.2011.10.001

[7] Rahaman, M.S.A., Ismail, A.F. and Mustafa, A. (2007) A Review of Heat Treatment on Polyacrylonitrile Fiber. Polymer Degradation and Stability, 92, 1421-1432. http://dx.doi.org/10.1016/j.polymdegradstab.2007.03.023

[8] Bajaj, P. and Roopanwal, A.K. (1997) Thermal Stabilization of Acrylic Precursors for the Production of Carbon Fibers: An Overview. Journal of Macromolecular Science, Part C: Polymer Reviews, 37, 97-147. http://dx.doi.org/10.1080/15321799708014734

[9] Sen, K., Bajaj, P. and Sreekumar, T.V. (2003) Thermal Behavior of Drawn Acrylic Fibers. Journal of Polymer Science Part B: Polymer Physics, 41, 2949-2958. http://dx.doi.org/10.1002/polb.10609

[10] Sun, T., Hou, Y. and Wang, H. (2009) Effect of Atmospheres on Stabilization of Polyacrylonitrile Fibers. Journal of Macromolecular Science, Part A, 46, 807-815.

[11] Jain, M.K. and Abhiraman, A.S. (1987) Conversion of Acrylonitrile-Based Precursor Fibres to Carbon Fibres: Part 1 A Review of the Physical and Morphological Aspects. Journal of Materials Science, 22, 278-300. http://dx.doi.org/10.1007/BF01160584

[12] Fitzer, E., Frohs, W. and Heine, M. (1986) Optimization of Stabilization and Carbonization Treatment of PAN Fibres and Structural Characterization of the Resulting Carbon Fibres. Carbon, 24, 387-395. http://dx.doi.org/10.1016/0008-6223(86)90257-5

[13] Dalton, S., Heatley, F. and Budd, P.M. (1999) Thermal Stabilization of Polyacrylonitrile Fibres. Polymer, 40, 55315543. http://dx.doi.org/10.1016/S0032-3861(98)00778-2

[14] Gupta, A. and Harrison, I. (1996) New Aspects in the Oxidative Stabilization of PAN-Based Carbon Fibers. Carbon, 34, 1427-1445. http://dx.doi.org/10.1016/S0008-6223(96)00094-2

[15] Wang, P.H. (1998) Aspects on Prestretching of PAN Precursor: Shrinkage and Thermal Behavior. Journal of Applied Polymer Science, 67, 1185-1190. http://dx.doi.org/10.1002/(SICI)1097-4628(19980214)67:7<1185::AID-APP3>3.0.CO;2-C

[16] Gupta, A. and Harrison, I. (1997) New Aspects in the Oxidative Stabilization of PAN-Based Carbon Fibers: II. Carbon, 35, 809-818. http://dx.doi.org/10.1016/S0008-6223(97)00025-0

[17] Gupta, A.K., Paliwal, D.K. and Bajaj, P. (1991) Acrylic Precursors for Carbon Fibers. Journal of Macromolecular Science, Part C: Polymer Reviews, 31, 1-89. http://dx.doi.org/10.1080/15321799108021557

[18] Chen, J. and Harrison, I. (2002) Modification of Polyacrylonitrile (PAN) Carbon Fiber Precursor via Post-Spinning Plasticization and Stretching in Dimethyl Formamide (DMF). Carbon, 40, 25-45. http://dx.doi.org/10.1016/S0008-6223(01)00050-1

[19] Ouyang, Q., Cheng, L., Wang, H. and Li, K. (2008) Mechanism and Kinetics of the Stabilization Reactions of Itaconic Acid-Modified Polyacrylonitrile. Polymer Degradation and Stability, 93, 1415-1421. http://dx.doi.org/10.1016/j.polymdegradstab.2008.05.021

[20] Grassie, N. and McGuchan, R. (1971) Pyrolysis of Polyacrylonitrile and Related Polymers-III. Thermal Analysis of Preheated Polymers. European Polymer Journal, 7, 1357-1371. http://dx.doi.org/10.1016/0014-3057(71)90032-2

[21] Grassie, N. and McGuchan, R. (1971) Pyrolysis of Polyacrylonitrile and Related Polymers-II: The Effect of Sample Preparation on the Thermal Behaviour of Polyacrylonitrile. European Polymer Journal, 7, 1091-1104. http://dx.doi.org/10.1016/0014-3057(71)90141-8

[22] Surianarayanan, M., Uchida, T. and Wakakura, M. (1998) Evolved Gases by Simultaneous TG-MS Technique and Associated Thermal Hazard in Drying of Polyacrylonitrile. Journal of Loss Prevention in the Process Industries, 11, 99-108. http://dx.doi.org/10.1016/S0950-4230(97)00032-6

[23] Tsuchiya, Y. and Sumi, K. (1977) Thermal Decomposition Products of Polyacrylonitrile. Journal of Applied Polymer Science, 21, 975-980. http://dx.doi.org/10.1002/app.1977.070210411 